\title{
AN ANALYTICAL ITERATIVE STATISTICAL ALGORITHM FOR IMAGE RECONSTRUCTION FROM PROJECTIONS
}

\author{
ROBERT CIERNIAK \\ Institute of Computational Intelligence \\ Częstochowa University of Technology, ul. Armii Krajowej 36, 42-200 Częstochowa, Poland \\ e-mail: cierniak@kik.pcz.czest.pl
}

\begin{abstract}
The main purpose of the paper is to present a statistical model-based iterative approach to the problem of image reconstruction from projections. This originally formulated reconstruction algorithm is based on a maximum likelihood method with an objective adjusted to the probability distribution of measured signals obtained from an x-ray computed tomograph with parallel beam geometry. Various forms of objectives are tested. Experimental results show that an objective that is exactly tailored statistically yields the best results, and that the proposed reconstruction algorithm reconstructs an image with better quality than a conventional algorithm with convolution and back-projection.
\end{abstract}

Keywords: computed tomography, image reconstruction from projections, statistical reconstruction algorithm.

\section{Introduction}

A key problem in computed tomography is the reconstruction of the image from projections. In this medical imaging system, the projections are obtained from an x-ray scanner classified by its geometry as one of the existing generations. There are several reconstruction methods presented in the literature to solve this problem. Among the most popular methods are analytical reconstruction algorithms based on convolution and back-projection operations (Lewitt, 1983). The Algebraic Reconstruction Technique (ART) has also been extensively explored (Kaczmarz, 1937) and applied (Thibault et al., 2007) in the past. One can say that all of the recent, practically applicable reconstruction algorithms can be assigned to one of these two methodologies of reconstructed image processing.

Currently, statistical reconstruction methods, such as the Maximum Likelihood (ML) approach and a development of this concept, the Maximum A Posterio$r i$ (MAP) estimation, are presented in the literature as being more robust and flexible than analytical inversion methods because they allow accurate modeling of the statistics of the projection data (Sauer and Bouman, 1993; Bouman and Sauer, 1996; Thibault et al., 2007). The MAP model has been incorporated in the construction of the Model-Based Iterative Reconstruction (MBIR) algorithm through application of the Iterative Coordinate Descent
(ICD) reconstruction method (the ICD reconstruction method as described comprehensively by Thibault et al. (2007), and the Non-Homogeneous ICD (NH-ICD) as presented by Zhou et al. (2011) are based on the MAP methodology). Because the signals obtained are adapted to the specific statistics for a given technique, the new algorithm yields a reduction in radiation dose during human body examination, as in the Adaptive Statistical Iterative Reconstruction (ASIR) algorithm (see, e.g., Silva et al., 2010; Yanagawa et al., 2010; Ren et al., 2012).

ICD is essentially an algebraic reconstruction technique where the image reconstruction consists of an iterative optimization process. It is worth emphasizing at this point that the ART reconstruction problem is very easy to reformulate into an optimization one. That is why it seems natural to use this technique for reconstruction algorithms based on the MAP (and, theoretically, the ML) model. The reconstruction algorithm proposed in this paper, owing to its analytical origins, avoids most of the difficulties connected with the use of the ART methodology, namely, it is very easy to establish the coefficients of the forward model in our method and the reconstruction process can be performed for every cross-section image separately, in contrast to the ART methodology, where calculations are performed for all the voxels in the range of the reconstructed 3D image (see, e.g., Thibault et al., 2007; DeMan and Basu, 2004).

In this paper, we present a reconstruction method 
which is based on an optimization process. Two forms of the objective of this optimization are established, which are derived based on the statistics of projection data obtained using transmission tomographic techniques (e.g., CT). We propose a modification of these functions so as to limit the analytical statistical model to the ML scheme. In the proposed reconstruction algorithm, we formulate a shift invariant system that allows us to easily decrease the computational complexity by transformation of the calculation performed in the frequency domain. A preliminary concept of this kind of image reconstruction from projections strategy has been represented in the literature only in the original works published by the author of this paper, for parallel scanner geometry (e.g., Cierniak, 2006; 2008a; 2008b) and for fan-beam geometry (e.g., Cierniak, 2009). The reconstruction algorithm schemes shown in these works are two-dimensional. This means that the actual reconstruction process is performed in a $2 \mathrm{D}$ variable space representing a deblurred image.

\section{Image reconstruction using parallel-beam projections}

The proposed reconstruction algorithm is based on parallel beam tomography (see also Cierniak, 2008a; 2008b). This method can be classified as an analytical approach and superficially resembles, but only in the order in which the operations are performed, the so-called $\rho$-filtered layergram method. A general view of our new iterative reconstruction algorithm is presented in Fig. 1

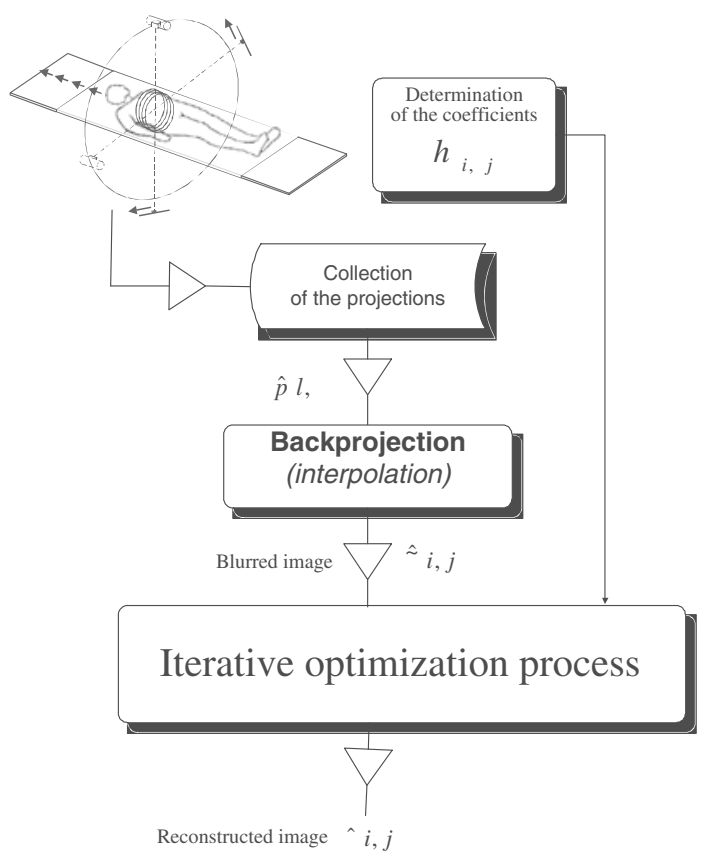

Fig. 1. General view of the reconstruction algorithm.

According to the scheme of the reconstruction algorithm shown in Fig. 1, the first step of the reconstruction procedure is a back-projection operation for a full revolution of the scanner. This operation is expressed as follows:

$$
\tilde{\mu}(x, y)=\int_{-\pi}^{\pi} p(s, \alpha) \mathrm{d} \alpha,
$$

where the function $\tilde{\mu}(x, y)$ denotes the image obtained after the back-projection operation, and $p(s, \alpha)$ is a projection determined by measuring the $\mathrm{x}$-ray intensity at a distance $s$ from the origin when a projection is made at a specific angle $\alpha$, as depicted in Fig. 2. The main

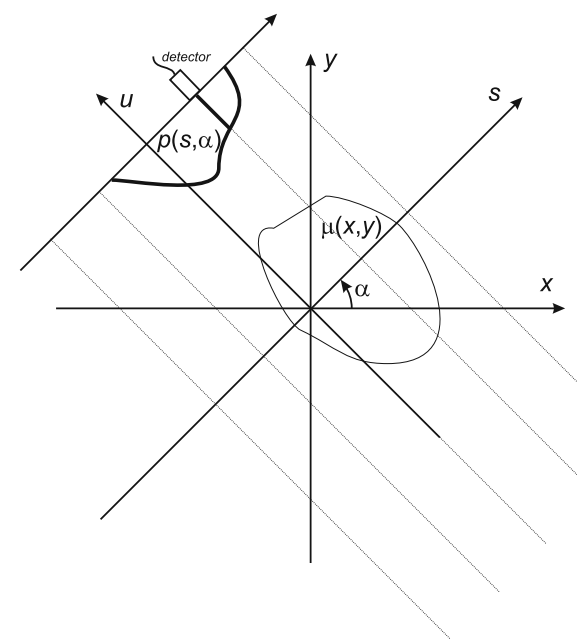

Fig. 2. Single projection.

purpose of the reconstruction process is to obtain the function $\mu(x, y)$, which denotes the unknown original image representing a cross-section of an examined body, using projections obtained from measurements carried out by the scanner.

It is worth noting that in practical implementations we consider the discrete form of the image $\hat{\mu}(i, j)$, where $i=1, \ldots, I$ and $j=1, \ldots, J$, and perform only a limited number of projections $p(s, \alpha)$ at a given angle $\alpha$. This number of projections is determined by the number of $\mathrm{x}$-ray detectors placed on the screen (these particular places can be designated by $s_{l}$, where $l=-L / 2, \ldots, L / 2$ and $L$ is an even number of detectors) and by the number of projection angles $\alpha_{\psi}$ ( $\psi$ is the index of the discrete projection angles, and $\psi=0, \ldots, \Psi-1$, where $\Psi$ is the total number of angles of projection performed). In the simplest case, this could be an equiangular distribution of intervals, where $\alpha_{\psi}=\psi \Delta_{\alpha}, \psi=0, \ldots, \Psi-1$ and the angular space between performed projections is $\Delta_{\alpha}=2 \pi / \Psi$. Thus, it is highly likely that for any given projection angle no ray passes through the pixel $(i, j)$ of the reconstructed image, and so it will be necessary to apply interpolation to obtain the appropriate projection 
during the back-projection operation. The operation can be expressed as follows:

$$
\bar{p}(s, \alpha)=\int_{-\infty}^{+\infty} p(\bar{s}, \alpha) \operatorname{int}(x \cos \alpha+y \sin \alpha-\bar{s}) \mathrm{d} \bar{s},
$$

where $p(s, \alpha)$ are projections obtained by the scanner, $\bar{p}(s, \alpha)$ are interpolated projections and $\operatorname{int}(\Delta s)$ is an interpolation function.

Thus, we have to replace $p(s, \alpha)$ in the formula (1) by $\bar{p}(s, \alpha)$ from the relation (2). In the standard direct approach, the image $\tilde{\mu}(x, y)$, obtained through the back-projection operation, includes information about the reconstructed image $\mu(x, y)$ which is strongly blurred by a geometrical term according to the following relation (Jain, 1989):

$$
\tilde{\mu}(x, y)=\int_{-\infty}^{+\infty} \int_{-\infty}^{+\infty} \mu(\bar{x}, \bar{y}) \cdot h^{\mathrm{dir}}(x-\bar{x}, y-\bar{y}) \mathrm{d} \bar{x} \mathrm{~d} \bar{y}
$$

where

$$
h^{\operatorname{dir}}(\Delta x, \Delta y)=\left((x-\bar{x})^{2}+(y-\bar{y})^{2}\right)^{-\frac{1}{2}},
$$

where kernel $h^{\text {dir }}$ is defined with the exception of the points $(\bar{x}, \bar{y})$, for which $x=\bar{x} \wedge y=\bar{y}$.

In our approach, the reconstruction from the projection operation is carried out by the direct recovery of this image from the blurred image $\tilde{\mu}(x, y)$ using an optimization method, where the reconstruction problem is based on the following relation, formulated originally by us (see, e.g., Cierniak, 2009)

$$
\tilde{\mu}(x, y)=\int_{-\infty}^{+\infty} \int_{-\infty}^{+\infty} \mu(\bar{x}, \bar{y}) h^{\mathrm{int}}(x-\bar{x}, y-\bar{y}) \mathrm{d} \bar{x} \mathrm{~d} \bar{y},
$$

where

$$
h^{\mathrm{int}}(\Delta x, \Delta y)=\int_{-\pi}^{\pi} \operatorname{int}(x \cos \alpha+y \sin \alpha) \mathrm{d} \alpha .
$$

Taking into account the discrete form of the feasible reconstruction procedure, we can present both relations (3) and (5) as follows:

$$
\hat{\tilde{\mu}}(i, j) \approx \sum_{\bar{i}} \sum_{\bar{j}} \hat{\mu}(\bar{i}, \bar{j}) \cdot h_{\Delta i, \Delta j}
$$

where the coefficients $h_{\Delta i, \Delta j}$ are

$$
\begin{aligned}
& h_{\Delta i, \Delta j}^{\mathrm{dir}} \\
& =\left\{\begin{array}{l}
\sum_{\psi=0}^{\Psi-1} \frac{\Delta_{\alpha}\left(\Delta_{s}\right)^{2}}{\sqrt{\left(\Delta i \Delta_{s} \cos \psi \Delta_{\alpha}\right)^{2}+\left(\Delta j \Delta_{s} \sin \psi \Delta_{\alpha}\right)^{2}}} \\
\text { if } i \neq \bar{i} \text { or } j \neq \bar{j}, \\
2 \pi \text { if } i=\bar{i} \text { and } j=\bar{j},
\end{array}\right.
\end{aligned}
$$

if we take into consideration the form (4), and

$$
\begin{aligned}
h_{\Delta i, \Delta j}^{\mathrm{int}}=\Delta_{\alpha}\left(\Delta_{s}\right)^{2} \sum_{\psi=0}^{\Psi-1} \operatorname{int}( & \Delta i \Delta_{s} \cos \psi \Delta_{\alpha} \\
& \left.+\Delta_{j} \Delta_{s} \sin \psi \Delta_{\alpha}\right),
\end{aligned}
$$

if we consider the proposed form (6).

The formula (7) defines the 2D discrete approximate reconstruction problem for the case of an equiangular distribution of projection angles. When a form of interpolation function used in the back-projection operation is taken into account (i.e., coefficients $h_{\Delta i, \Delta j}$ are determined according to the formula (9)), we obtain an accurate model. This model is used as a starting point for further discussion because it allows us to formulate an ML approach to the reconstruction problem. Simulations have shown that the use of the relation (8) leads to very poor results.

In the discrete realization of our reconstruction method, using the strategy of projection acquisition presented above and based on Eqn. (1), the image obtained after the back-projection operation can be presented as follows:

$$
\hat{\tilde{\mu}}(i, j) \cong \Delta_{\alpha} \sum_{\psi=0}^{\Psi-1} \bar{p}\left(s_{i j}, \psi \Delta_{\alpha}\right)
$$

where

$$
s_{i j}=i \Delta_{s} \cos \psi \Delta_{\alpha}+j \Delta_{s} \sin \psi \Delta_{\alpha}
$$

is the coordinate defining the position of point $(i, j)$ on the screen, during a projection carried out at angle $\alpha_{\psi}=$ $\psi \Delta_{\alpha}$. Additionally, we take into account the discrete form of Eqn. (2),

$$
\bar{p}\left(s_{i j}, \psi \Delta_{\alpha}\right)=\Delta_{s} \sum_{l} p\left(l \Delta_{s}, \psi \Delta_{\alpha}\right) \operatorname{int}\left(s_{i j}-l \Delta_{s}\right) .
$$

The coefficients $h_{\Delta i, \Delta j}$ are determined only once, before the reconstruction process is started, according to the formula (9). Computer simulations performed by us have shown that it is necessary to increase the number $\Psi$ 
of simulated projections during this process, multiplying it by an integer $k$, whose value is chosen experimentally. This operation extends the set of simulated projection angles to a new range, namely, to $\alpha_{\psi}=\psi \Delta_{\alpha} / k$, where $\psi=0, \ldots, k \Psi-1$, and now the coefficients $h_{\Delta i, \Delta j}$ are calculated in the following way:

$$
\begin{array}{r}
h_{\Delta i, \Delta j} \cong\left(\Delta_{s}\right)^{2} \frac{\Delta_{\alpha}}{k} \sum_{\psi=0}^{k \Psi-1} \operatorname{int}\left(\Delta i \Delta_{s} \cos \psi \frac{\Delta_{\alpha}}{k}\right. \\
\left.+\Delta j \Delta_{s} \sin \psi \frac{\Delta_{\alpha}}{k}\right) .
\end{array}
$$

It is worth stressing that this modification does not have excessive costs, because these values of $h_{\Delta i, \Delta j}$ are pre-calculated, and their results can be fixed permanently for all further reconstruction processes. These properties could make our approach very attractive when implementing the reconstruction algorithm presented here. Owing to these low computational real costs, an analysis of the number of views that need to be performed during the calculation of the $h_{\Delta i, \Delta j}$ coefficients is not crucial. However, it has been shown previously (see, e.g., Cierniak, 2011) that the parameter $k$ should be sufficiently large, e.g., $k=10$. Regarding the form of the interpolation function, computer simulations have proved that, from among the forms of this function considered, the use of linear interpolation provided the best results.

It is expected that matrices of the coefficients $h_{\Delta i, \Delta j}$ are similar for all pixels of the reconstructed image. Thus, we can make a simplification and determine these coefficients for only pixel $(i, j)=(0,0)$, assuming that

$$
\begin{aligned}
h_{\Delta i, \Delta j} \cong & h_{i, j} \\
= & \left(\Delta_{s}\right)^{2} \frac{\Delta_{\alpha}}{k} \sum_{\psi=0}^{k \Psi-1} \operatorname{int}\left(i \Delta_{s} \cos \psi \frac{\Delta_{\alpha}}{k}\right. \\
& \left.+j \Delta_{s} \sin \psi \frac{\Delta_{\alpha}}{k}\right) .
\end{aligned}
$$

\section{Statistical considerations for the problem of image reconstruction from projections}

Currently, the most important challenge in the field of computed tomography is concerned with the statistical considerations of the signals in reconstruction algorithms (see, e.g., Thibault et al., 2007; Xu and Tsui, 2009). Our statistical iterative reconstruction algorithm is based on the probabilistic model of the physical phenomena present in the $\mathrm{x}$-ray measurement system. For the purpose of constructing a new statistical approach to the reconstruction problem, we will reformulate the statistical conception of the ML strategy for image reconstruction as presented, e.g., by Sauer and Bouman $(1993 ; 1992)$ or Thibault et al. (2007).
We will start the derivation of our statistical reconstruction method with the introduction of one of the fundamental quantities associated with $\mathrm{x}$-ray radiation-the intensity $I$ of x-rays. This is proportional to the number of photons $n$ passing through unit area in unit time. Therefore, we can write that

$$
I \propto n .
$$

If we examine a sample of material (such as the human body) using $\mathrm{x}$-rays, we obtain the following intensity, registered by the $\mathrm{x}$-ray detector on the other side of the sample:

$$
I(U)=I(0) \cdot \mathrm{e}^{-\int_{0}^{U} \mu(x, y) \mathrm{d} u}
$$

where $I(0)$ is the initial x-ray intensity, $I(U)$ is the x-ray intensity after passing through a distance $U$.

Finding the logarithm of both the sides of Eqn. (15), we obtain a quantity, called the projection, which has fundamental significance for image reconstruction algorithms. In addition, in order to obtain an image of the cross-section of an object in the plane of the projection, we need to identify parameters for the quantity $p$, as shown in Fig. 2. According to the relation (15), we can write

$$
p(s, \alpha)=\ln \left(\frac{I(0)}{I(U)}\right)=\ln \left(\frac{n_{0}}{n_{s, \alpha}}\right),
$$

where $n_{0}$ is the initial number of x-ray photons (we assume that $n_{0}$ is the same for all projections), $n_{s, \alpha}$ is the number of $\mathrm{x}$-ray photons after passing through the distance $U$. In statistical approaches, the number $n_{s, \alpha}$ of photons registered by a detector at a given projection angle is represented by a random variable $N_{s, \psi}$. It is a generally accepted assumption that $N_{s, \psi}$ represents a Poisson-distributed random variable. Thus, the probability of registering $n_{s, \psi} \mathrm{x}$-ray photons at the detector is the following:

$$
\operatorname{Pr}\left(N_{s, \psi}=n_{s, \psi}\right)=\frac{n_{s, \alpha}^{*} n_{s, \alpha}}{n_{s, \alpha} !} \mathrm{e}^{-n_{s, \alpha}^{*}},
$$

where $n_{s, \alpha}^{*}$ is the expected value of $N_{s, \alpha}$.

The following log form of the probability described by (17) is often used (it is only a rescaling of this expression) (see, e.g., Sauer and Bouman, 1993):

$$
\begin{aligned}
L 1\left(n_{s, \alpha}\right) & =\ln \left(P\left(N=n_{s, \alpha}\right)\right) \\
& =n_{s, \alpha} \ln \left(n_{s, \alpha}^{*}\right)-\ln \left(n_{s, \alpha} !\right)-n_{s, \alpha}^{*} .
\end{aligned}
$$

If we use Stirling's approximation of $\ln n_{\psi}$ ! for $n_{\psi} \gg 0$,

$$
\ln \left(n_{s, \alpha} !\right) \approx n_{s, \alpha} \ln \left(n_{s, \alpha}\right)-n_{s, \alpha},
$$

then we obtain a formula for evaluating the error in emission tomography,

$$
L 2\left(n_{s, \alpha}\right)=n_{s, \alpha} \ln \left(\frac{n_{s, \alpha}^{*}}{n_{s, \alpha}}\right)+n_{s, \alpha}-n_{s, \alpha}^{*} .
$$


The above form of divergence could be a very interesting starting point for the formulation of reconstruction algorithms dedicated to emission tomography techniques. However, x-ray computed tomography, which we are considering here, is a transmission tomography and therefore we can take into account the relations

$$
n_{s, \alpha}=n_{0} \mathrm{e}^{-p(s, \alpha)}
$$

and

$$
n_{s, \alpha}^{*}=n_{0} \mathrm{e}^{-p^{*}(s, \alpha)},
$$

which come directly from the definition (16), in the relation (20). In this way we can derive the following formula for the evaluation of the error in transmission tomography:

$$
\begin{aligned}
& L 3(p(s, \alpha)) \\
& \qquad \begin{array}{l}
\quad n_{0} \mathrm{e}^{-p(s, \alpha)}\left(p(s, \alpha)-p^{*}(s, \alpha)\right) \\
\quad+n_{0} \mathrm{e}^{-p(s, \alpha)}-n_{0} \mathrm{e}^{-p^{*}(s, \alpha)} .
\end{array}
\end{aligned}
$$

Expanding the term $n_{0} \mathrm{e}^{-p^{*}(s, \alpha)}$ in a second-order Taylor series around $p(s, \alpha)$, where $\Delta p=p^{*}(s, \alpha)-p(s, \alpha)$, we can obtain the following approximation (for details, see Appendix):

$$
L 4(p(s, \alpha))=-\frac{1}{2} n_{0} \mathrm{e}^{-p(s, \alpha)}\left(p^{*}(s, \alpha)-p(s, \alpha)\right)^{2},
$$

where

$$
p^{*}(s, \alpha)=\ln \left(\frac{n_{0}}{n_{s, \alpha}^{*}}\right)
$$

can be interpreted as the expected value of the projection measurement.

The relation (24) describes the statistical nature of only one projection in x-ray computed tomography, i.e., that registered by a specific x-ray detector at a fixed distance $s$ from the origin of the scanner and at a specific projection angle $\alpha$. In the reconstruction algorithm proposed by us, the reconstruction procedure begins with the back-projection operation, as shown by Eqn. (10). This can be written as follows:

$$
p_{\Sigma}(i, j)=\Delta_{\alpha} \sum_{\psi=0}^{\Psi-1} p\left(s_{i j}, \alpha_{\psi}\right)
$$

where $s_{i j}=i \Delta_{s} \cos \alpha_{\psi}+j \Delta_{s} \sin \alpha_{\psi}$ is the coordinate defining the position of point $(i, j)$ on the screen, during a projection carried out at angle $\alpha_{\psi}$.

On the other hand, the back-projection operation, based on real measurements, results in a blurred image, and, regarding the relation (10), we can assign

$$
p_{\Sigma}(i, j)=\hat{\tilde{\mu}}(i, j) \text {. }
$$

It is easy to interpret our reconstruction method as a search for an expected attenuation value in a cross-section of the examined body to match the reconstructed image. We are then fully justified in stating that if we were to detect an expected projection value on the screen, we would deal with it as if it were an expected value of the attenuation coefficient inside the observed object. By analogy with the relation (27), taking into account the properties of the back-projection operation and the basic formula (7), we can write the following:

$$
p_{\Sigma}^{*}(i, j)=\hat{\tilde{\mu}}^{*}(i, j) \cong \sum_{\bar{i}} \sum_{\bar{j}} \hat{\mu}^{*}(\bar{i}, \bar{j}) \cdot h_{\Delta i, \Delta j},
$$

where $\hat{\mu}^{*}(i, j)$ is the expected value of attenuation coefficients $\hat{\mu}(i, j)$.

Accordingly, taking into account the error measure (24), fundamental for x-ray tomography, we can derive an appropriate loss function for the algorithm presented in this paper,

$$
\begin{aligned}
L 5 & =-\frac{1}{2} n_{0} \mathrm{e}^{-p_{\Sigma}(i, j)}\left(p_{\Sigma}^{*}(i, j)-p_{\Sigma}(s, \alpha)\right)^{2} \\
& =-\frac{1}{2} n_{0} \mathrm{e}^{-\hat{\tilde{\mu}}(i, j)}\left(\hat{\tilde{\mu}}^{*}(i, j)-\hat{\tilde{\mu}}(i, j)\right)^{2} .
\end{aligned}
$$

The loss function $L 5$ from the relation 29) takes into account only one pixel $(i, j)$ from the reconstructed image. To take into account all $I \cdot J$ pixels in the reconstructed image, it is necessary to extend the error measure (29) as a summation of the participation of all the pixels, each in part expressed by (29). Taken all together this gives us

$$
L 6=-\frac{n_{0}}{2} \sum_{i=1}^{I} \sum_{j=1}^{\mathrm{J}} \frac{1}{\sigma^{2}(i, j)}(e(i, j))^{2}
$$

where

$$
e(i, j)=\sum_{\bar{i}} \sum_{\bar{j}} \hat{\mu}^{*}(\bar{i}, \bar{j}) \cdot h_{\Delta i, \Delta j}-\hat{\tilde{\mu}}(i, j)
$$

and

$$
\sigma^{2}(i, j)=\frac{1}{\sqrt{\mathrm{e}^{p\left(s_{i j}, 0\right)}+\mathrm{e}^{p\left(s_{i j}, 1\right)}+\cdots+\mathrm{e}^{p\left(s_{i j}, \Psi-1\right)}}} .
$$

Taking into account the observation that the projections $p\left(s_{i j}, \psi\right)$ are close to each other for a specific pixel $(i, j)$, we can simplify the relation 32 to the following form:

$$
\sigma^{2}(i, j)=\sqrt{\frac{\mathrm{e}^{-p\left(s_{i j}, 0\right)}}{\Psi}} .
$$

But if we have to use a reconstruction methodology based on a pure ML scheme, without the regularizing a prio$r i$ term, we have to prevent certain instabilities of the reconstruction process which arise in this case. To do this, 


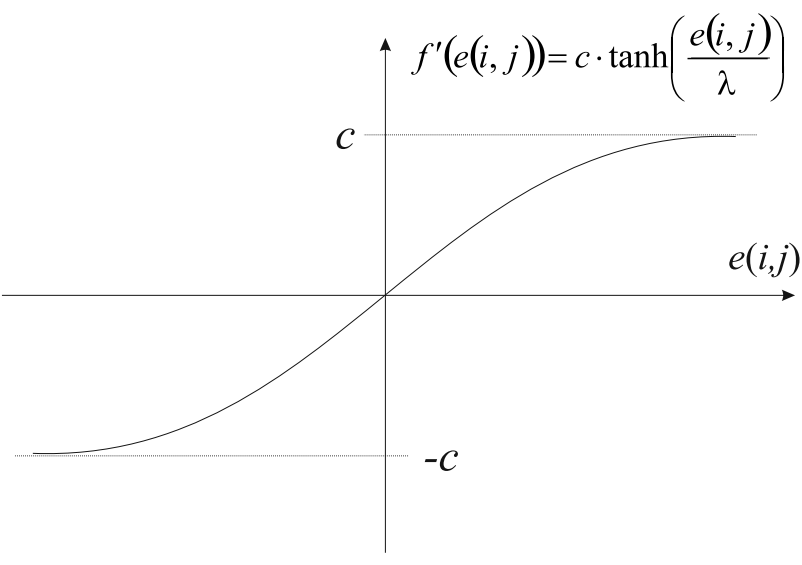

Fig. 3. Form of the derivative of the function 35 .

we propose the modifying criterion 30 and replacing the loss function $L 6$ with the following error measure:

$$
\begin{aligned}
L 7= & -\frac{n_{0}}{2} \sum_{i=1}^{I} \sum_{j=1}^{\mathrm{J}} \frac{1}{\sigma(i, j)} \\
& \cdot f\left(\sum_{\bar{i}} \sum_{\bar{j}} \hat{\mu}^{*}(\bar{i}, \bar{j}) \cdot h_{\Delta i, \Delta j}-\hat{\tilde{\mu}}(i, j)\right),
\end{aligned}
$$

where we propose the following form of the function $f(\cdot)$ :

$$
f(e(i, j))=\lambda^{2} \cdot \ln \cosh \left(\frac{e(i, j)}{\lambda}\right)
$$

where $\lambda$ is a slope coefficient.

It is worth stressing that the derivative of the function (35) has the well-known form of the hyperbolic tangent function, namely,

$$
f^{\prime}(e(i, j))=c \cdot \tanh \left(\frac{e(i, j)}{\lambda}\right)=c \frac{1-\mathrm{e}^{-e(i, j) / \lambda}}{1+\mathrm{e}^{-e(i, j) / \lambda}}
$$

presented graphically in Fig. 3

The probability measure (34) can be used to formulate a maximum-likelihood estimation method (see, e.g., Sauer and Bouman, 1992). We will manipulate the values of $\mu^{*}(i, j)$ so as to maximize the divergence defined in Eqn. (34). This procedure can be written mathematically as

$$
\mu_{\max }^{*}=\arg \max _{\mu^{*}}(L 7) .
$$

It is easy to see that (37) is an optimization problem and, as usually in this case, can be easily transformed into a more convenient form for finding the minimum

$$
\begin{aligned}
& \mu_{\min }^{*} \\
& =\arg \min _{\mu^{*}}\left(\frac{n_{0}}{2} \sum_{i=1}^{\mathrm{I}} \sum_{j=1}^{\mathrm{J}} \frac{1}{\sigma^{2}(i, j)}\right. \\
& \left.\quad f\left(\sum_{\bar{i}} \sum_{\bar{j}} \hat{\mu}^{*}(\bar{i}, \bar{j}) \cdot h_{\Delta i, \Delta j}-\hat{\tilde{\mu}}(i, j)\right)\right) .
\end{aligned}
$$

We propose the gradient descent method to solve the optimization problem described by the formula (38) because of its simplicity. Contrary to other possible optimization algorithms, such as the Newton-Raphson or the Levenberg-Marquard methods, this method is much less time consuming, and at the same time fast enough in converging to the solution. In this case, the pixels in the reconstructed image will take values according to the following very easy iterative procedure:

$$
\begin{aligned}
& \mu^{*(t+1)}(i, j) \\
& =\mu^{*(t)}(i, j) \\
& \quad-\sum_{\bar{i}=1}^{I} \sum_{\bar{j}=1}^{J} \frac{1}{\sigma_{\Sigma}^{2}(\bar{i}, \bar{j})} f^{\prime}\left(e^{(t)}(\bar{i}, \bar{j})\right) h_{\triangle i, \Delta j},
\end{aligned}
$$

where $f^{\prime}$ is a function from (36) and, based on (31),

$$
e^{(t)}(\bar{i}, \bar{j})=\sum_{i} \sum_{j} \hat{\mu}^{*(t)}(i, j) \cdot h_{\Delta i, \Delta j}-\hat{\tilde{\mu}}(\bar{i}, \bar{j}) .
$$

\begin{tabular}{|c|c|c|c|c|c|c|}
\hline \multirow[t]{2}{*}{ No. } & \multicolumn{2}{|c|}{$\begin{array}{l}\text { Coordinates } \\
\text { of the centre }\end{array}$} & \multirow{2}{*}{$\begin{array}{c}\text { Semi-major } \\
\text { axis } \\
a\end{array}$} & \multirow{2}{*}{$\begin{array}{c}\text { Semi-minor } \\
\text { axis } \\
b\end{array}$} & \multirow{2}{*}{$\begin{array}{l}\text { Angle } \\
\text { of rot. } \\
\alpha_{0}\left[{ }^{\circ}\right]\end{array}$} & \multirow{2}{*}{$\begin{array}{c}\mu \\
10^{-2}\end{array}$} \\
\hline & $x_{0}$ & $y_{0}$ & & & & \\
\hline 1 & $\overline{0.00}$ & $\overline{0.0000}$ & $\overline{\overline{0}}$ & $\overline{\overline{0.9}}$ & $\overline{\overline{0.0}}$ & $\overline{\overline{2.00}}$ \\
\hline II & 0.00 & -0 . & & & 0.0 & -0.98 \\
\hline III & 0.22 & 000 & & 0.3 & -18.0 & -0.02 \\
\hline IV & -0.22 & 0.00 & 0 & 0.4 & 18.0 & -0.02 \\
\hline V & 0.00 & & 0 & 0.2 & 0.0 & 0.01 \\
\hline VI & 00 & & & 0.0 & 0.0 & 0.01 \\
\hline VII & 0 & -0 . & & & 0.0 & 0.01 \\
\hline VIII & -0.08 & & & & 0.0 & 0.01 \\
\hline IX & 0.00 & -0.6050 & 0.0230 & 0.0230 & 0.0 & 0.01 \\
\hline $\mathrm{X}$ & 0.06 & -0.6050 & 0.0230 & 0.0460 & 0.0 & 0.01 \\
\hline
\end{tabular}

\section{Experimental results}

In our experiments, we adapted the well-known Shepp-Logan phantom of the head. We proposed a mathematical model of a head consisting of relevant ellipses given by Shepp and Logan (1974), as depicted in Table 1

Because we are only simulating a statistical estimation of the projections, all the values of the

Table 1. Parameters of the ellipses making up the mathematical model of the head phantom. 


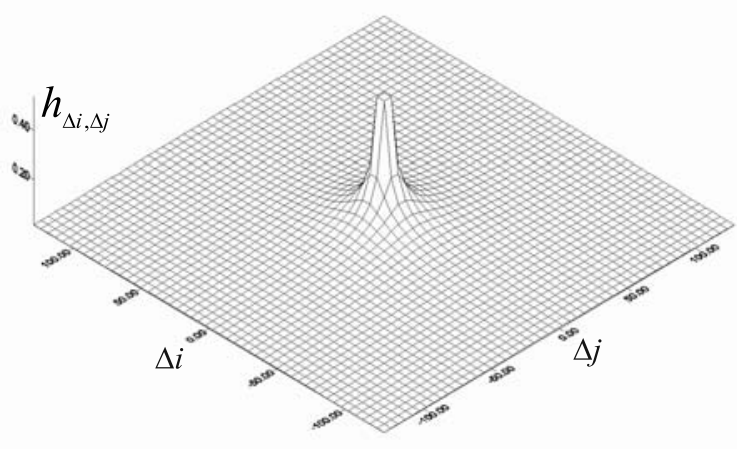

Fig. 4. Values of coefficients $h_{\Delta i, \Delta j}$.

attenuation coefficients placed in the last column of Table 1 were divided by a factor $10^{2}$ to facilitate the calculations. The model formed in this way was used to generate projections with a Poisson probability distribution. In every case we start our simulations from a calculation of the accurate values of the projection $p^{*}(s, \alpha)$ in the traditional way, and then values of radiation intensity $I^{*}$ are established according to the following relation:

$$
\ln \left(I^{*}(U)\right)=\ln (I(0))-p^{*}(s, \alpha),
$$

where $I(0)$ is the same initial $\mathrm{x}$-ray intensity for all projections (in our experiments $I(0)=10^{6}$ ).

After the determination of the $I^{*}$ value, we generated the statistical value of the intensity $I(U)$ with a Poisson probability distribution with the expected value $I^{*}(U)$, and using this value we back-calculated the projections $p(s, \alpha)$. These projections were used in our reconstruction algorithm.

In all our experiments, we fixed the following parameters: $L=170$ measurement points (detectors) on the screen, the number of the angles $\Psi=519$, and the size of the processed image $I \times J=128 \times 128$ pixels.

Coefficients $h_{\Delta i, \Delta j}$ were pre-computed using the relation 13 before we started the reconstruction process, and these coefficients were fixed for the subsequent processing. They were determined with the value $k=$ 1000, which was chosen experimentally. If, in Eqn. (13), we use the linear interpolation function, the values of the coefficients $h_{\Delta i, \Delta j}$ obtained are presented in Fig. 4

After the determination of the coefficients $h_{\Delta i, \Delta j}$, we can start the reconstruction procedure. Firstly, we perform the back-projection operation using the relationships (11) and (10) to get a blurred image of the $\mathrm{x}$-ray attenuation distribution in a given cross-section of the investigated object. The result of this operation is presented in Fig. 5 .

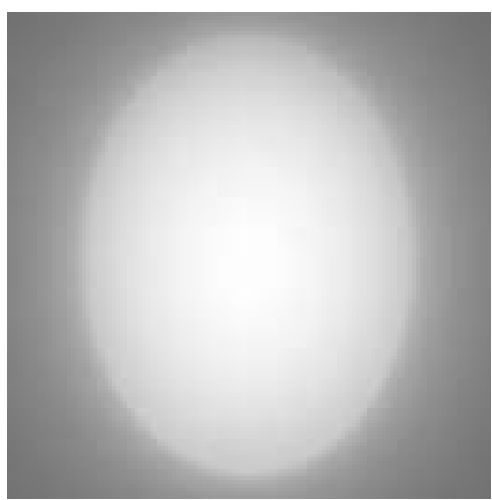

Fig. 5. Distorted image of the mathematical model obtained after the operation of back-projection.

Secondly, the iterative optimization process is performed. During this process, the optimization is carried out using a gradient descent method. The optimization procedure, being indeed a reconstruction process, is described by the relations (39) and (40), which are based on the objective $L 7$ described by (34). It is worth noting that we used $\lambda=10^{-2}$ and $c=3.33 \cdot 10^{-5}$, chosen experimentally. Other combinations of these values are possible, but with the constraint that expression $\lambda c$ should not exceed approximately $3.3 \cdot 10^{-7}$ to avoid instabilities during performance of the reconstruction process. Because the measure $L 7$ is a convex function, this optimization process converges to a unique solution.

Because we should not rely only on a subjective evaluation of the results of the reconstruction algorithm, we also used objective measures, i.e., the well-known $M S E$ and $S N R$ error measures defined as follows:

$$
\begin{gathered}
M S E=\frac{1}{I^{2}} \sum_{i=1}^{\mathrm{I}} \sum_{j=1}^{\mathrm{I}}\left(\hat{\mu}^{*(t)}(i, j)-\hat{\mu}(i, j)\right)^{2}, \\
S N R=10 \log _{10} \frac{\sum_{i=1}^{\mathrm{I}} \sum_{j=1}^{\mathrm{I}}(\hat{\mu}(i, j))^{2}}{\sum_{i=1}^{\mathrm{I}} \sum_{j=1}^{\mathrm{I}}\left(\hat{\mu}^{*}(t)(i, j)-\hat{\mu}(i, j)\right)^{2}},
\end{gathered}
$$

where $\hat{\mu}^{*(t)}(i, j)$ is the reconstructed image after $t$ iterations, $\hat{\mu}(i, j)$ is the original image of the Shepp-Logan mathematical phantom, and a new parameter proposed by us establishing the variation of the image in the region of the ellipse III (a completely smooth element), with the following form:

$$
V A R_{I I I}=\frac{\sum_{(i, j)_{I I I}}\left(\hat{\mu}^{*(t)}(i, j)-\hat{\mu}_{m_{I I I}}^{*(t)}\right)^{2}}{M_{I I I}},
$$

where $\hat{\mu}_{m_{I I I}}^{*(t)}$ is the mean value inside ellipse III in the reconstructed image after $t$ iterations, $\mathrm{M}_{I I I}$ is the number of pixels inside element III. 


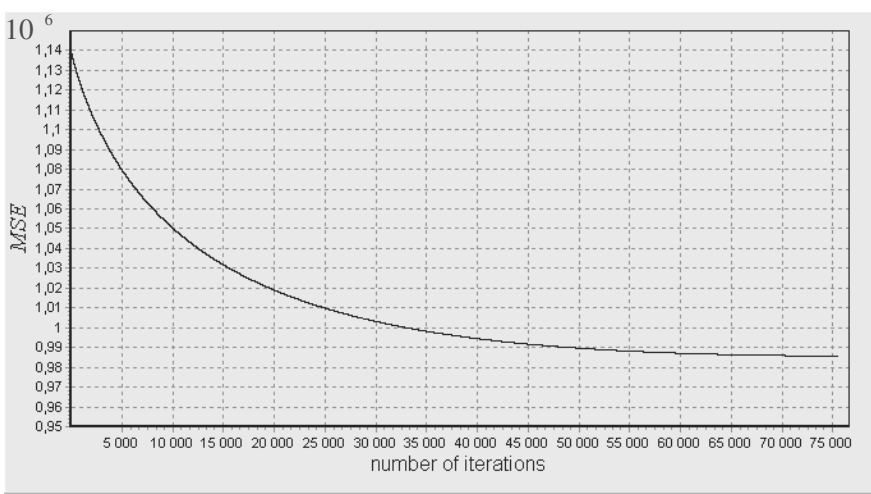

Fig. 6. Results of the reconstruction process dependent on the number of projections during the calculation of the reconstructed image, evaluated by the $M S E$ measure (see Eqn. (43) in the case of deterministic signals.

This parameter has to evaluate the ability of the reconstruction algorithm to decrease the influence of the noise present in the measurements of the image obtained in the reconstruction process. The smaller the parameter $V A R_{I I I}$ for the homogeneous region inside ellipse III, the greater our ability to perceive any possible details appearing in this area.

We can observe the results of the performed investigations in Fig. 6 for deterministic signals, and in Fig. 7 for signals in the presence of noise. Views of the reconstructed images of the mathematical phantom in the cross-section after $t$ iterations are presented in Fig. 8(c) when deterministic signals are taken into consideration, and in Figs. 9 and 10 (b), (c) for stochastic signals. One can compare the results obtained using the original phantom image (Fig. 8(a)) and the image reconstructed by a standard reconstruction method (Figs. 8(b), 9(a) and 10(a)) (Jain, 1989).

It is worth noting that the rim near the bones of the skull in the reconstructed image is caused, in our interpretation, by the overfitting effect. This phenomenon is strongly associated with places in the reconstructed image where our model expressed by (7) is the most inaccurate, i.e., at the edges (e.g., borders between bones and other tissues). The problem of how to prevent these artifacts will be further investigated. Probably, a way to avoid this effect in the reconstructed image is to accelerate the optimization process for pixels where the errors (31) are the largest (at the edges).

One can observe in Fig. 7 that errors $M S E$ and $V A R_{I I I}$ decrease quickly with the number of iterations specific for the given approach, and then both errors slowly increase after they have reached their minimums. We should stop the reconstruction process at this point because these error measures (especially parameter

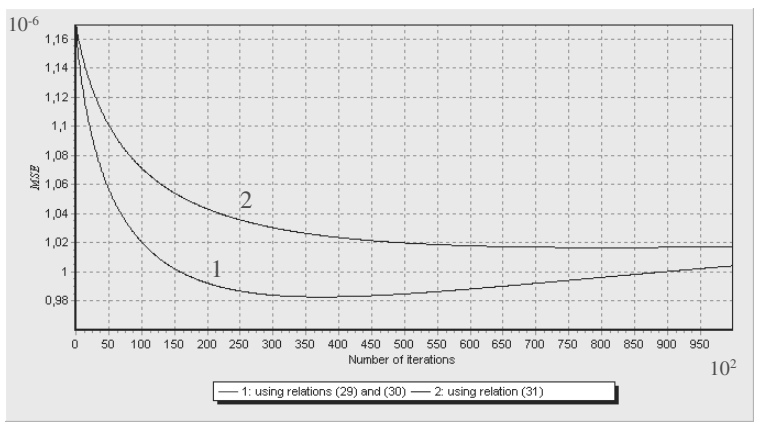

(a)

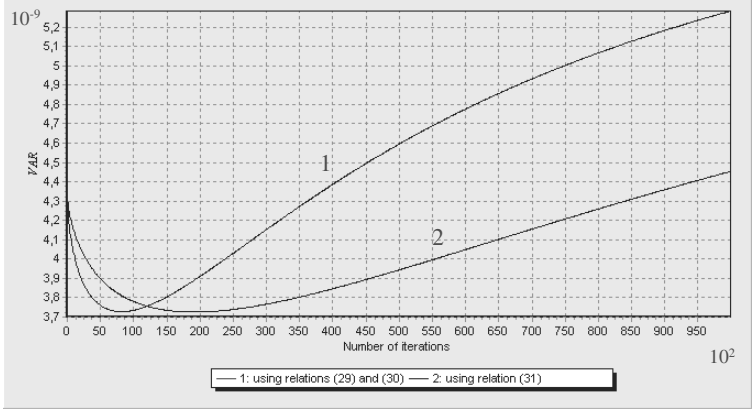

(b)

Fig. 7. Results of the reconstruction process dependent on the number of projections during the calculation of the reconstructed image, evaluated by the $M S E$ measure (a), the VAR $R_{I I I}$ measure (b), in the case of stochastic signals.

$V A R_{I I I}$ ) describe the quality of the reconstructed image in the regions of the investigated cross-section which are interesting from a diagnostic point of view.

\section{Conclusion}

The main goal of the presented paper was to present a statistical model-based iterative approach to the problem of image reconstruction from projections. In our approach, the formulation of the image reconstruction from projections problem is consistent with an analytical scheme of image processing defining the class of reconstruction algorithm. Accordingly, it is possible to avoid the fundamental difficulties associated with approaches based on the algebraic concept of the performed image processing. Generally, the reconstruction problem as formulated by us is very compact, and can be classified as a shift-invariant system. Elements of this system can be pre-calculated before we start the reconstruction procedure. Additionally, we showed how to make our approach an optimal reconstruction method which considers the statistical properties of the signals measured by a real $\mathrm{x}$-ray computed tomography scanner. A form of objective has been derived, which is optimal for our approach taking 


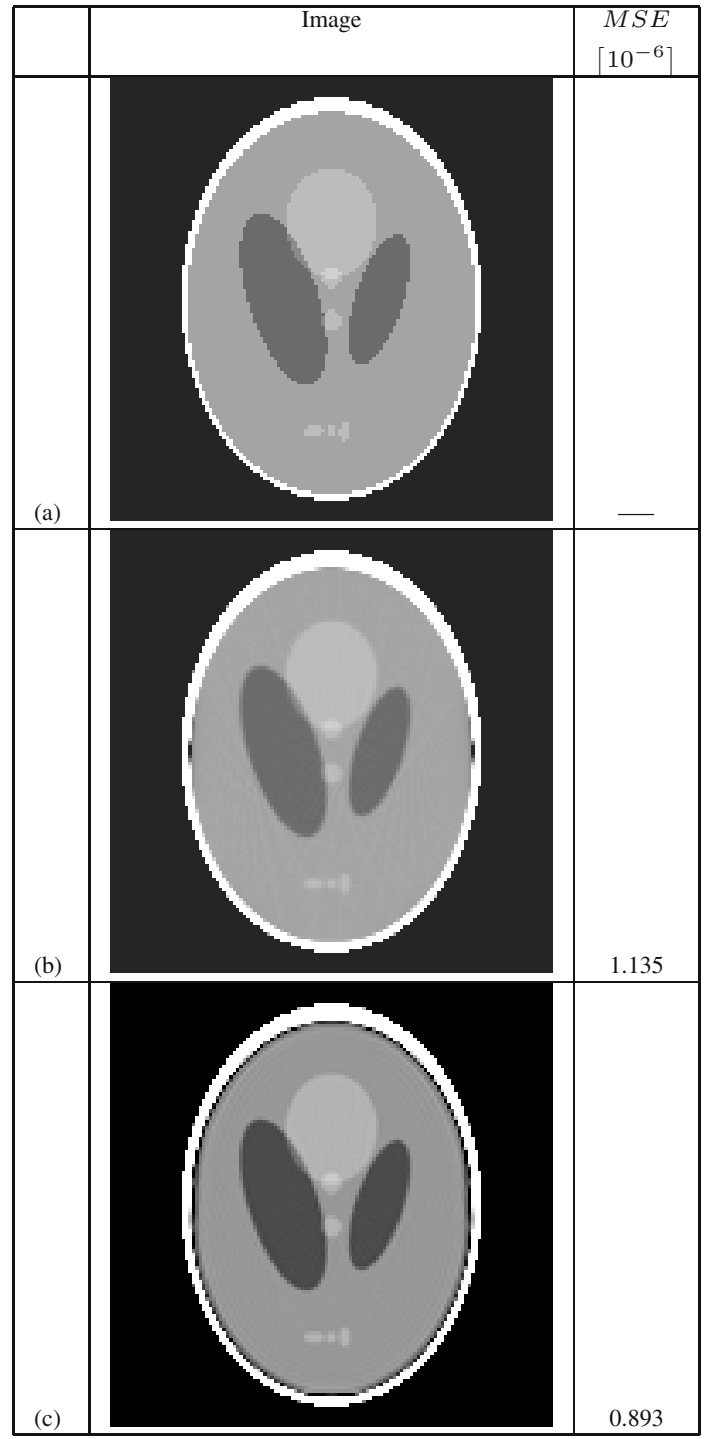

Fig. 8. View of the images (window: $C=0.0102, W=$ 0.0011) when the signals registered by the detectors are deterministic: original image (a), reconstructed image using the standard convolution/back-projection method with rebinning and the Shepp-Logan kernel (b), reconstructed image using the method described in this paper at $t=75000$ (c).

into account the statistical conditions. This objective is fundamental for the design of our iterative reconstruction procedure based on a maximum likelihood estimation method. Simulations were performed which show that our reconstruction procedure is stable without the introduction of any additional regularization term, in contrast to the maximum a posteriori probability estimation. During the computer simulations, the reconstructed image of the cross-section of a mathematical phantom was investigated. The reconstruction algorithm designed by us yields an image with high accuracy when compared with

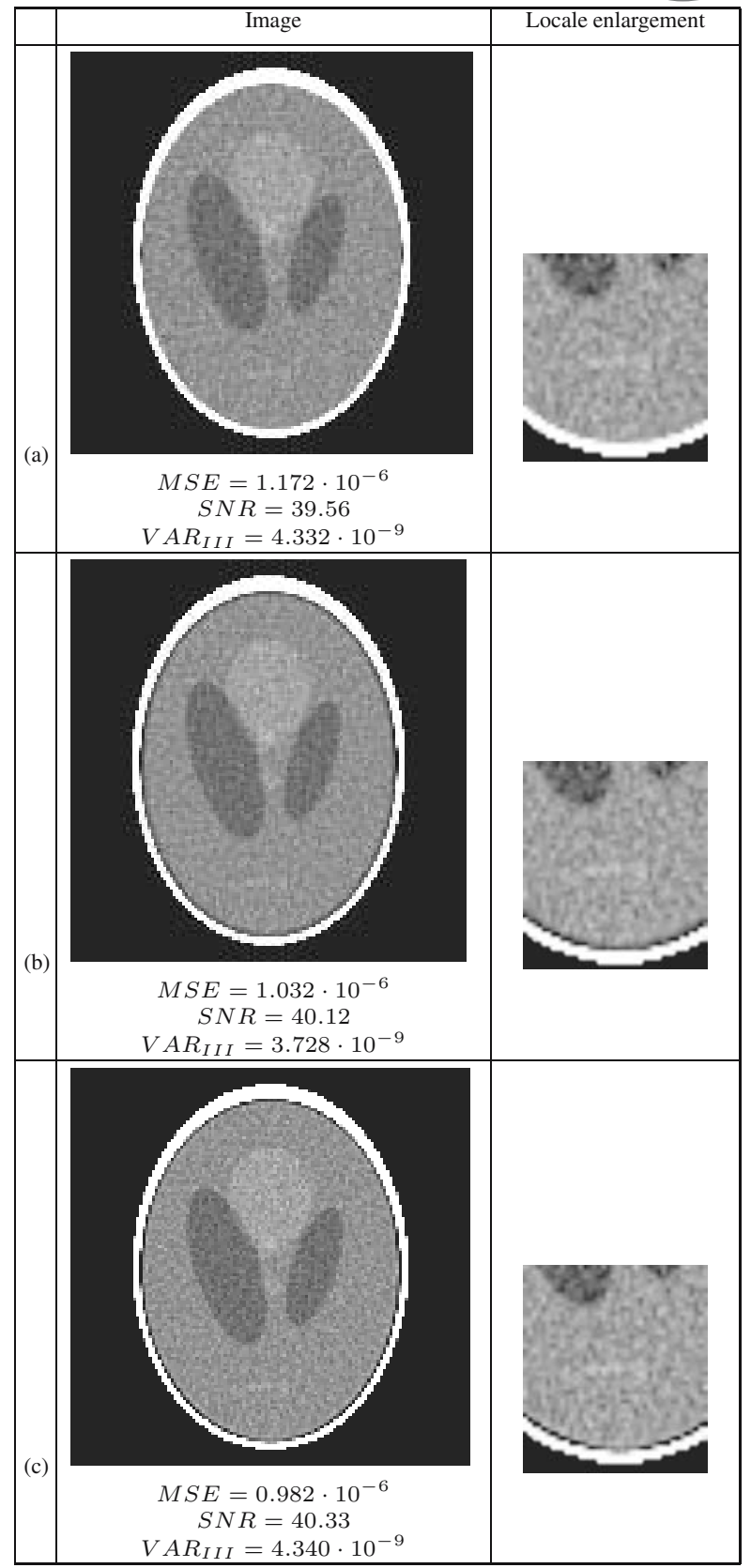

Fig. 9. View of the images (window: $C=0.0102, W=$ 0.0011 ) when the signals registered by the detectors are stochastic: reconstructed image using the standard convolution/back-projection method with rebinning and the Shepp-Logan kernel (a), reconstructed image using the method described in this paper at $t=8000(\mathrm{~b})$, and at $t=38000$ (c), using the relations (30) and 32.

the standard method, as measured objectively using the quality measures (43) and (44).

Thanks to the formulation of our reconstruction problem as a shift-invariant system we can decrease the computational complexity of our method by transformation of the performed calculation in the 


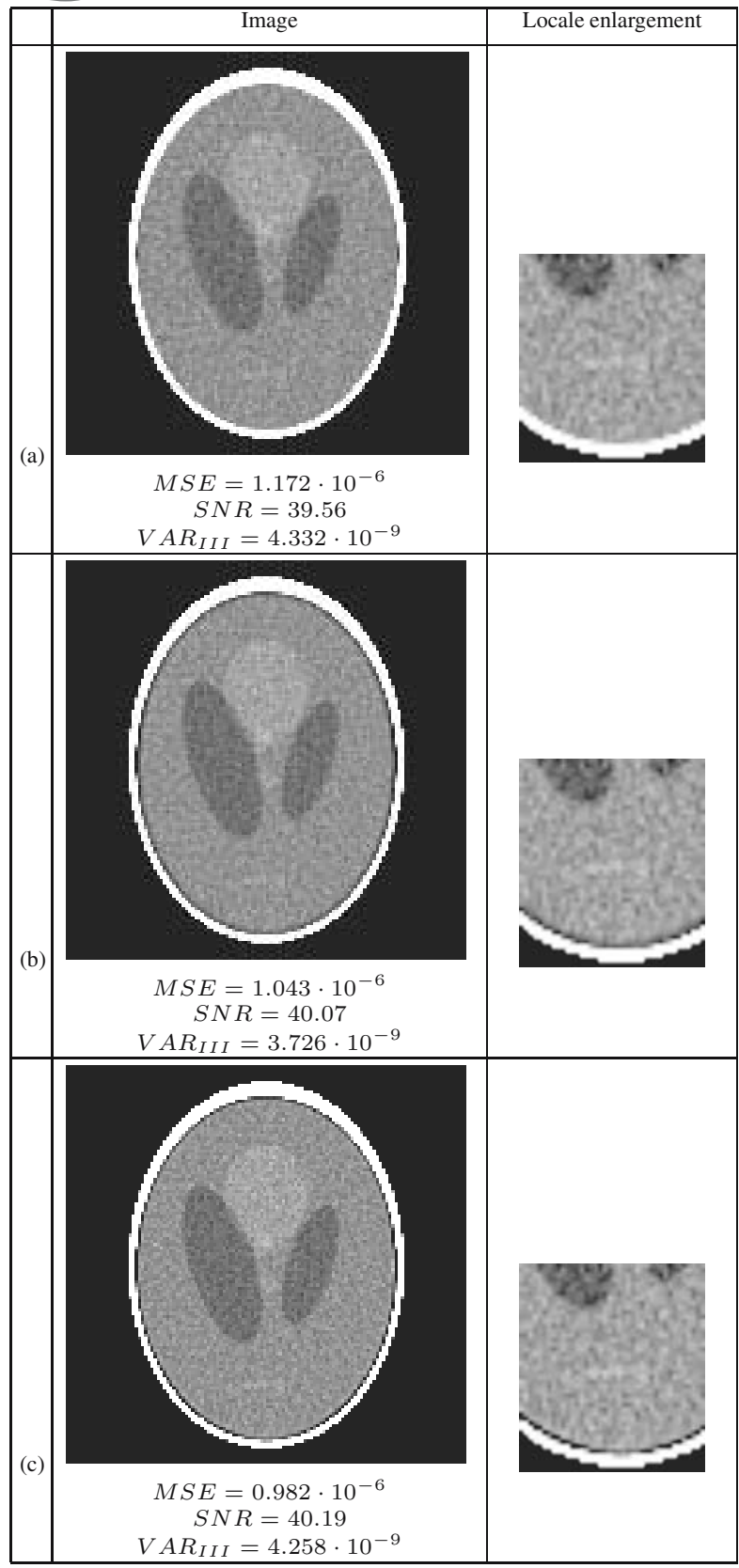

Fig. 10. View of the images (window: $C=0.0102, W=$ 0.0011 ) when the signals registered by the detectors are stochastic: reconstructed image using the standard convolution/back-projection method with rebinning and the Shepp-Logan kernel (a), reconstructed image using the method described in this paper at $t=$ 20000 (b), and at $t=80000$ (c), using the relations (30) and 33 .

frequency domain. This therefore reduces the number of operations of every iteration of the performed optimization process to the level of $\mathcal{O}\left(2 I^{2} \log _{2} I\right)$. In comparison with the algebraic approach represented by the ICD algorithm, where the computational complexity of every iteration of the optimization process is proportional to $I^{2} \times \Psi \times L$ (approximately $I^{4}$ ), this method produces a huge acceleration. Of course, our method is much slower than the standard convolution/back-projection algorithm, but that method does not have the property of adaptation to the statistical nature of the CT technique.

Having a solution to the image reconstruction from projections for parallel beams, we can extend our results to other projection geometries: fan-beams and cone-beams, in particular those incorporated in spiral tomography. Particularly, it was shown that it is possible to extend our results to techniques based on methods such as the SSR method (see, e.g., Noo et al., 1999; Bruder et al., 2000) or methods based on the principles of the ASSR algorithm (see, e.g., Kachelriss et al., 2000; 2001; 2004), as was presented by Cierniak (2010).

\section{References}

Bouman, C.A. and Sauer, K.D. (1996). A unified approach to statistical tomography using coordinate descent optimization, IEEE Transactions on Image Processing 5(3): 480-492.

Bruder, H., Kachelriess, M., Schaller, S., Stierstorfer, K. and Flohr, T. (2000). Single-slice rebinning reconstruction in spiral cone-beam computed tomography, IEEE Transactions on Medical Imaging 9(9): 873-887.

Cierniak, R. (2006). A novel approach to image reconstruction from projections using Hopfield-type neural network, in L. Rutkowski, R. Tadeusiewicz, L.A. Zadeh and J. Żurada (Eds.), Artificial Intelligence and Soft Computing, Lecture Notes in Artificial Intelligence Vol. 4029, Springer-Verlag, Berlin/Heidelberg, pp. 890-898.

Cierniak, R. (2008a). A new approach to image reconstruction from projections using a recurrent neural network, Artificial Intelligence in Medicine 43(2): 113-125.

Cierniak, R. (2008b). A new approach to image reconstruction from projections problem using a recurrent neural network, International Journal of Applied Mathematics and Computer Science 18(2): 147-157, DOI: 10.2478/v10006-008-0014-y.

Cierniak, R. (2009). New neural network algorithm for image reconstruction from fan-beam projections, Neurocomputing 72(13-15): 3238-3244.

Cierniak, R. (2010). A three-dimensional neural network based approach to the image reconstruction from projections problem, in L. Rutkowski, R. Scherer, R. Tadeusiewicz, L.A. Zadeh and J. Żurada (Eds.), Artificial Intelligence and Soft Computing, Lecture Notes in Artificial Intelligence, Vol. 6113, Springer-Verlag, Berlin/Heidelberg, pp. 505-514.

Cierniak, R. (2011). Neural network algorithm for image reconstruction using the grid-friendly projections, $A u$ stralasian Physical \& Engineering Sciences in Medicine 34(3): 375-389. 
DeMan, B. and Basu, S. (2004). Distance-driven projection and backprojection in three dimensions, Physics in Medicine and Biology 49(11): 2463-2475.

Jain, A.K. (1989). Fundamentals of Digitals Image Processing, Prentice-Hall, Englewood Cliffs, NJ.

Kachelriess, M., Fuchs, T. and Schaller, S. (2001). Advanced single-slice rebinning for tilted spiral cone-beam CT, Medical Physics 31(6): 1033-1041.

Kachelriess, M., Knaup, M. and Kalender, W.A. (2004). Extended parallel backprojection for standard three-dimensional and phase-correlated four-dimensional axial and spiral cone-beam CT with arbitrary pitch, arbitrary cone-angle, and $100 \%$ dose usage, Medical Physics 31(6): 1623-1641.

Kachelriess, M., Schaller, S. and Kalender, W.A. (2000). Advanced single-slice rebinning in cone-beam spiral CT, Medical Physics 27(4): 754-773.

Kaczmarz, S. (1937). Angenaeherte aufloesung von systemen linearer gleichungen, Bulletin International de l'Académie Polonaise des Sciences et des Lettres 35: 355-357.

Lewitt, R.M. (1983). Reconstruction algorithms: Transform methods, Proceedings of the IEEE 71(3): 390-408.

Noo, F., Defrise, M. and Clackdoyle, R. (1999). Single-slice rebinning method for helical cone-beam CT, Physics in Medicine and Biology 44(2): 561-570.

Ren, Q., Dewan, S.K., Li, M., Li, J., Mao, D., Wang, Z. and Hua, Y. (2012). Comparison of adaptive statistical iterative and filtered back projection reconstruction techniques in brain CT, European Journal of Radiology 81(10): 2597-2601.

Sauer, K. and Bouman, C. (1992). Bayesian estimation of transmission tomograms using segmentation based optimization, IEEE Transactions on Nuclear Science 39(4): 1144-1152.

Sauer, K.D. and Bouman, C.A. (1993). A local update strategy for iterative reconstruction from projections, IEEE Transactions on Signal Processing 41(3): 480-492.

Shepp, L.A. and Logan, B.F. (1974). The Fourier reconstruction of a head section, IEEE Transactions on Nuclear Science NS-21: 21-43.

Silva, A.C., Lawder, H.J., Hara, A., Kujak, J. and Pavlicek, W. (2010). Innovations in CT dose reduction strategy: Application of the adaptive statistical iterative reconstruction algorithm, American Journal of Roentgenology 194(1): 191-199.

Thibault, J.B., Sauer, K.D., Bouman, C.A. and Hsieh, J. (2007). A three-dimensional statistical approach to improved image quality for multislice helical CT, Medical Physics 34(11): 4526-4544.

Xu, J. and Tsui, B.M.W. (2009). Electronic noise modeling in statistical iterative reconstruction, IEEE Transactions on Image Processing 18(6): 1228-1238.

Yanagawa, M., Honda, O., Yoshida, S., Kikuyama, A., Inoue, A., Sumikawa, H., Koyama, M. and Tomiyama, N. (2010). Adaptive statistical iterative reconstruction technique for pulmonary CT: Image quality of the cadeveric lung on standard- and reduced-dose CT, Academic Radiology 17(10): 1259-1266.

Zhou, Y., Thibault, J.B., Bouman, C.A., Sauer, K.D. and Hsieh, J. (2011). Fast model-based x-ray CT reconstruction using spatially non-homogeneous ICD optimization, IEEE Transactions on Image Processing 20(1): 161-175.

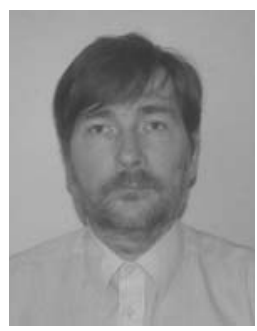

Robert Cierniak received an M.S. degree in electronic engineering in 1990, and a Ph.D. degree in computer science in 1995 , both from the Cracow University of Technology, Poland. From 1991 to 2013 he conducted research at the Częstochowa University of Technology, Poland. From 1991 to 1996 he was with the Institute of Electronics and Control Systems, and in the period from 1996 to 2013 with the Institute of Computational Intelligence. He has recently become a full professor at this institute. His main interests include neural networks, biomedical applications of artificial intelligence, computer vision and image processing.

\section{Appendix}

The derivation of the objective $L 4$ can be initialized by returning to the relation (24),

$$
\begin{aligned}
& L 3(p(s, \alpha)) \\
& \quad=n_{0} \mathrm{e}^{-p(s, \alpha)}\left(p(s, \alpha)-p^{*}(s, \alpha)\right) \\
& \quad+n_{0} \mathrm{e}^{-p(s, \alpha)}-n_{0} \mathrm{e}^{-p^{*}(s, \alpha)} .
\end{aligned}
$$

If we expand the term $\mathrm{e}^{-p^{*}(s, \alpha)}$ in a second-order Taylor series around $p(s, \alpha)$, where $\Delta p=p^{*}(s, \alpha)-p(s, \alpha)$, we can obtain the following approximation:

$$
\begin{aligned}
& \mathrm{e}^{-p^{*}(s, \alpha)} \\
& \cong \mathrm{e}^{-p(s, \alpha)}-\mathrm{e}^{-p(s, \alpha)}\left(p^{*}(s, \alpha)-p(s, \alpha)\right) \\
& \quad+\frac{1}{2} \mathrm{e}^{-p(s, \alpha)}\left(p^{*}(s, \alpha)-p(s, \alpha)\right)^{2} .
\end{aligned}
$$

Taking the above approximation into account in the relation $\mathrm{A} 1 \mathrm{~b}$, we can perform the following derivation:

$$
\begin{aligned}
L 3 & (p(s, \alpha)) \\
\cong & L 4(p(s, \alpha)) \\
& +n_{0} \mathrm{e}^{-p(s, \alpha)}\left(p(s, \alpha)-p^{*}(s, \alpha)\right)+n_{0} \mathrm{e}^{-p(s, \alpha)} \\
& -n_{0} \mathrm{e}^{-p(s, \alpha)}-n_{0} \mathrm{e}^{-p(s, \alpha)}\left(p(s, \alpha)-p^{*}(s, \alpha)\right) \\
& -\frac{1}{2} n_{0} \mathrm{e}^{-p(s, \alpha)}\left(p^{*}(s, \alpha)-p(s, \alpha)\right)^{2} \\
= & -\frac{1}{2} n_{0} \mathrm{e}^{-p(s, \alpha)}\left(p^{*}(s, \alpha)-p(s, \alpha)\right)^{2} .
\end{aligned}
$$

Received: 14 March 2013

Revised: 13 June 2013

Re-revised: 10 July 2013 